\title{
Use of diffuse reflections in tunable diode laser absorption spectroscopy: implications of laser speckle for gas absorption measurements
}

\author{
D Masiyano, J Hodgkinson and, R P Tatam \\ Engineering Photonics Group, Cranfield University, Cranfield, Bedfordshire, MK43 \\ OAL, UK \\ r.p.tatam@cranfield.ac.uk
}

\begin{abstract}
The use of diffusely scattering materials as a means of eliminating interference fringes has been investigated. Their use introduces laser speckle that can contribute a random, rather than periodic, uncertainty to gas measurements. We have established a method for quantifying the uncertainty due to speckle and investigated ways of reducing it. We characterised the speckle at $823 \mathrm{~nm}$ allowing the use of low cost CCD cameras. We have tested the principle of the model by making gas absorption measurements on the $1650 \mathrm{~nm}$ methane absorption line using wavelength modulation spectroscopy, for which preliminary results are presented.
\end{abstract}

\section{Introduction}

The advantages of using tunable diode laser absorption spectroscopy (TDLAS) for trace gas sensing include; fast response times, high sensitivity and high target gas selectivity. However, the sensitivity of many practical TDLAS systems is limited by the formation of unintentional Fabry-Perot interference fringes in the optical path between the source and detector[1]. The spacing between the maxima of these fringes can be in the same wavelength range as Doppler and pressure-broadened molecular line widths $\left(10^{-3}-10^{-2} \mathrm{~cm}^{-1}\right)$ [2]; this can lead to distortion or complete obscuring of the shape and strength of the absorption line[3] (Figure 1(c) and 3(b)). Thermal and mechanical instabilities mean that the fringes can not be removed by simple subtraction techniques. The formation of fringes is shown in Figure 1. 

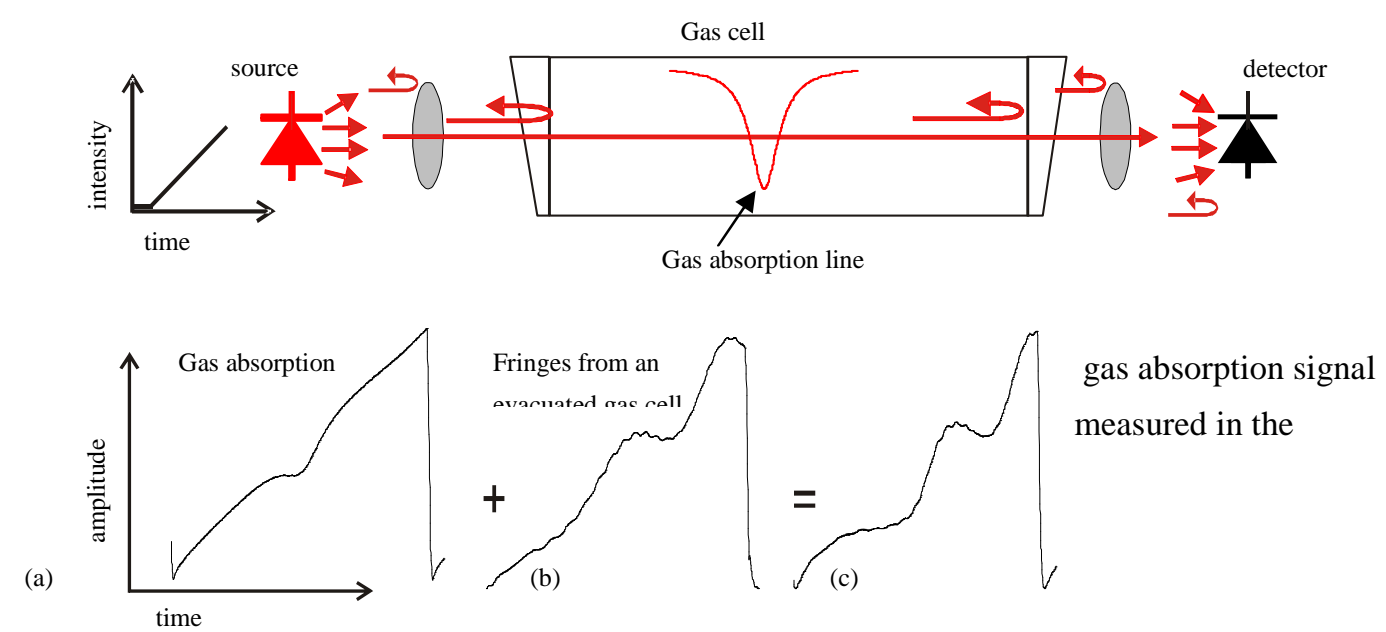

Figure 1. Illustration of formation of interference fringes in direct absorption spectroscopy: reflections from laser windows, detector windows, lenses, and gas cell windows can lead to the formation of interference fringes. (a) gas absorption signal measured with no interference effects. (b) interference fringes from an evacuated gas cell (c) gas absorption obscured by interference fringe signals.

Design techniques to reduce etalon formation include; the use of optical isolators, use of reflective rather than refractive optics, angling and antireflection coating of reflective surfaces[4]. Techniques for eliminating or reducing the amplitude of the fringe signal include: (a) mechanically oscillating the path length with external devices[1,5,6], (b) introduction of an asynchronous current in addition to the usual modulation current through the laser diode[7], (c) use of alternative waveforms[8,9] and (d) postdetection filtering[10]. The disadvantage of method (a) is that the detection bandwidth is limited by the mechanical motion and signal integration that is required[11]. Methods (b) and (c) are less effective and tend to wash out the gas absorption signal when the free spectral range (FSR) is comparable to the gas absorption linewidth[2]. Sun and Whittaker[11] have demonstrated a variation of method (b) that is effective for a wide range of FSR to absorption line width ratios. Post detection filtering can only be applied where the signal and the interference spectra are distinguishable in the frequency domain[2].

In general the alignment of the optical components is critical. This often leads to complex designs with tight tolerances on optical component alignment, and can therefore be difficult and expensive to maintain in field instruments.

Here, we present an alternative approach based on the use of diffuse reflections, known in some circumstances to reduce interference fringes[12]. However, their use introduces laser speckle that can contribute an intensity uncertainty to gas detection measurements. This research aims to (a) investigate the deliberate use of diffuse reflections as a means of eliminating interference fringes and (b) quantify the intensity uncertainty due to laser speckle and investigate methods of reducing it. We present preliminary results of a systematic study of these effects. 
Our analysis draws heavily from the field of speckle and electronic speckle interferometry; therefore we begin with a brief introduction to speckle fundamentals. Ennos[13] and Jones and Wykes[14] have given a more comprehensive statistical analysis of speckle.

\section{Speckle theory}

Monochromatic light incident on an optically rough surface is scattered in random directions and the surrounding region is filled with a speckle pattern formed by the interference of the reflected components (Figure 2).

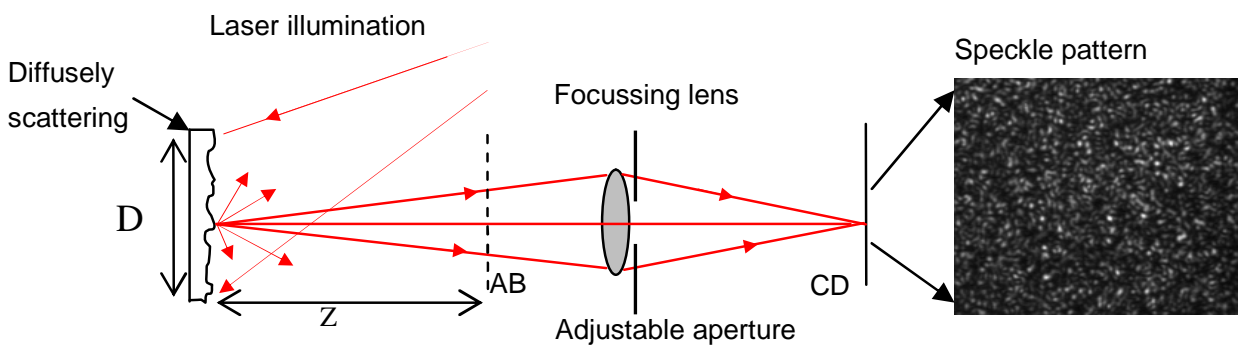

Figure 2. Illustration of the formation of speckle. Key, D; the illuminated area, Z; the distance to the observation plane $\mathrm{AB}, \mathrm{CD}$; the image plane

The lateral size $\varepsilon$ of the individual speckles at the screen $\mathrm{AB}\left(\varepsilon_{0}\right.$, objective speckle) and $\mathrm{CD}\left(\varepsilon_{\mathrm{s}}\right.$ subjective speckle) in Figure 2 are given by

$$
\begin{gathered}
\varepsilon_{o}=1.22 \lambda Z / D \\
\varepsilon_{s}=1.22 \lambda(1+\Pi) F \#
\end{gathered}
$$

where $\mathrm{D}$ is the illuminated area, $\mathrm{F} \#$ is the ratio of the focal length to aperture and $\Pi$ the magnification at which the lens is operating[13]. The scattered field amplitudes are randomly distributed and therefore the intensity values follow a negative exponential distribution

$$
P(I)=\frac{1}{\langle I\rangle} \exp (-I /\langle I\rangle)
$$

with the important consequence that its standard deviation $\sigma$ equals the mean[15]. The normalised standard deviation or contrast $C$ of a polarised speckle pattern is given by $C=\sigma /\langle I\rangle$. The contrast of a fully developed static speckle pattern is therefore always equal to unity. Adding $M$ uncorrelated speckle patterns on an irradiance basis results in suppression of speckle contrast to $C=\sigma / \sqrt{M}\langle I\rangle[15]$. 
The level of speckle intensity noise on a detector of size $\mathrm{d} x \mathrm{~d}$ is related to the number of speckles $\mathrm{N}$ and therefore the speckle size $\varepsilon$. The number of speckles can be approximated by $d^{2} / \varepsilon^{2}$.We expect the level of speckle-related intensity noise, $\Delta I / I$, for a single speckle field to be given by $1 / \sqrt{N}$ or $\varepsilon / d$. From the above considerations, the speckle related uncertainty can be conveniently written in terms of (2) as

$$
\frac{\Delta I}{I}=\frac{1.22 \lambda(1+\Pi) F \#}{\mathrm{~d}}
$$

\section{TDLAS}

The basic principle of TDLAS, its applications and comparisons of the different TDLAS techniques have been described elsewhere in the literature[16]. For our investigations, we have chosen to employ wavelength modulation spectroscopy (WMS), but the general principle applies to all TDLAS modulation schemes. In WMS, the laser wavelength is modulated by varying the laser drive current at a frequency of several kilohertz. The laser wavelength can be simultaneously scanned across a single gas absorption line by a ramp signal at a frequency lower than the modulation frequency typically several Hertz. The detected signal is then demodulated by a phase sensitive detector at the modulating frequency or its harmonics. The principle of WMS is illustrated in Figure 3.

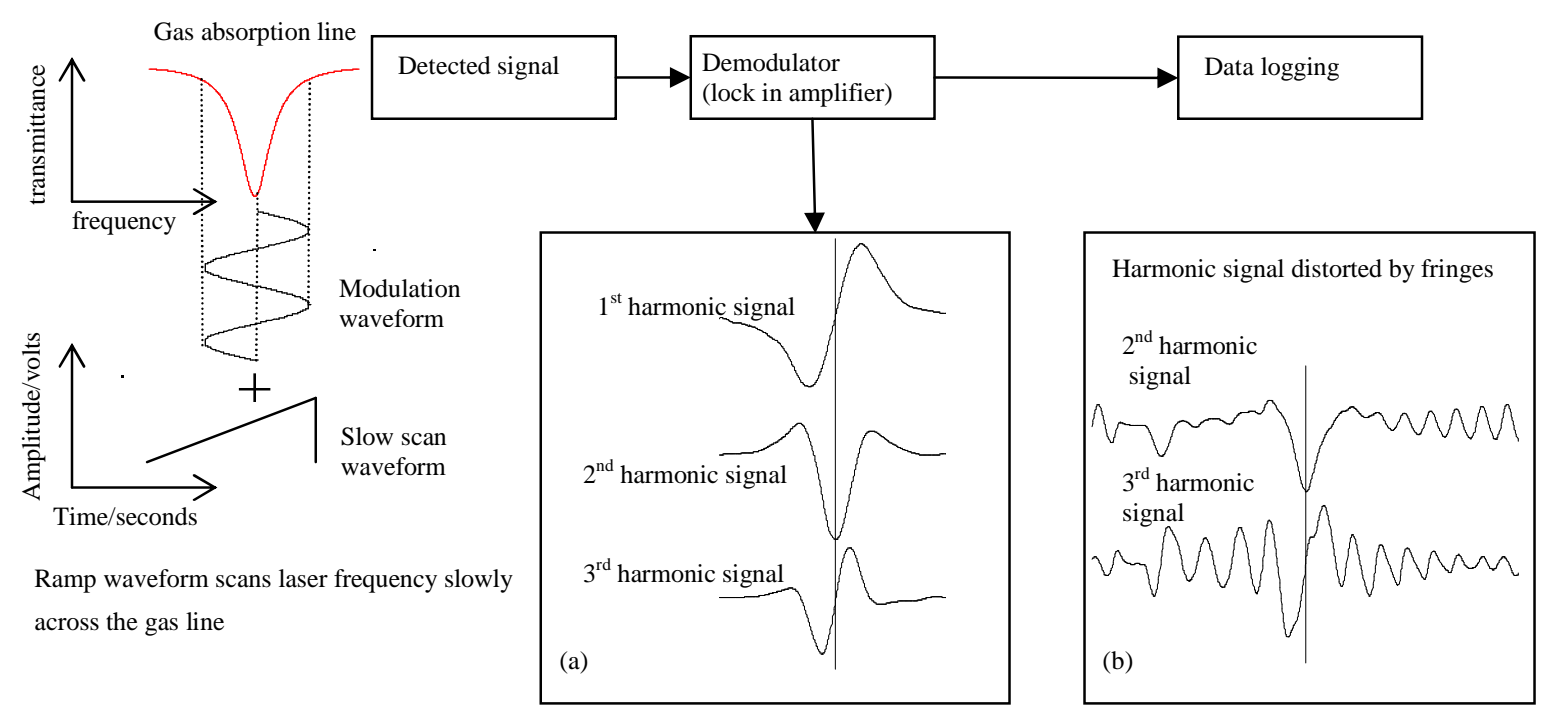

Figure 3: Illustration of the principle of WMS. (a) harmonic signals generated in the absence of interference fringes, (b) harmonic signals distorted by interference fringes.

The use of phase sensitive detection leads to a significant improvement in signal to noise ratio by reducing the measurement bandwidth. In addition, detection at higher harmonics shifts the operation to higher frequencies, at which laser excess noise is reduced and intensity modulation removed. The WMS set up for methane detection at $1651 \mathrm{~nm}$ is described in section 7.1 and illustrated in Figure 14. 


\section{Use of diffuse reflections in TDLAS}

We designed a model of the WMS setup at $823 \mathrm{~nm}$ so we could view the speckle with a silicon CCD camera. The experimental configuration shown in Figure 4 included: (i) a tunable diode laser driven so as to simulate TDLAS based gas detection, (ii) various geometries (including reflection and transmission types) of a simulated gas cell containing a diffusely scattering surface in combination with one of a number of different types of window (a wedged window is illustrated) and (iii) an interrogation system employing a silicon CCD camera. We used single mode optical fibre for beam delivery where it was more convenient to do so. The fibre based setup is described below.

\subsection{Experimental setup}

A $150 \mathrm{~mW}$ single longitudinal mode laser diode (Spectra Diode Labs 5400 series) emitting at $823 \mathrm{~nm}$ was coupled into a singlemode fibre with angle cleaved ( 8 degrees) end faces via a Faraday isolator. The isolator served to prevent feedback into the laser cavity which could have detrimental effects on the optical characteristics of the diode. The laser operating current and temperature were controlled by Profile LDC 202 and TED 200 controllers respectively. The light was divided by a 50:50 coupler. One arm was passed to a Fabry Perot scanning interferometer (Tec-Optics FPI25) for monitoring the laser diode emission wavelength. The other arm was directed to the gas cell model via a beam splitter. A calibrated variable aperture was used at the imaging lens L3 to control the $F \#$ of the system. This determines the size of the speckles at the detector array according to equation (2). The light from the gas cell was detected by a CCD camera (Pearpoint P176, 8 bit camera with $11.5 \mu \mathrm{m} \times 13 \mu \mathrm{m}$ pixels). The video signal from the camera was transferred to a frame grabbing card (National Instruments IMAQ PCI 1408) housed in a computer running Labview ${ }^{\mathrm{TM}}$ software. We could use either the CCD camera or a detector as the sensor for quantitative analysis of the speckle related uncertainty. The CCD camera was used for quantitative analysis only for speckle sizes $\varepsilon$ comparable or larger than the pixel sizes $r_{p}$. For $\varepsilon \ll<r_{p}$, the detector was used as the sensor for quantitative analysis and the camera for alignment and qualitative analysis only. This was done to maintain the validity of the statistics used. The detector was mounted on a micrometer $x y z$ translator that has a resolution of $50 \mu \mathrm{m}$ and translation of $13 \mathrm{~mm}$ in all axes. We used amplified detectors with variable gain of 3 different sizes (Thorlabs PDA400; 0.8 $\mathrm{mm}^{2}$, PDA55; $12.96 \mathrm{~mm}^{2}$, and PDA50B; $19.6 \mathrm{~mm}^{2}$ ). The detector output was fed to a lock in amplifier (Stanford Research Systems SR850). 


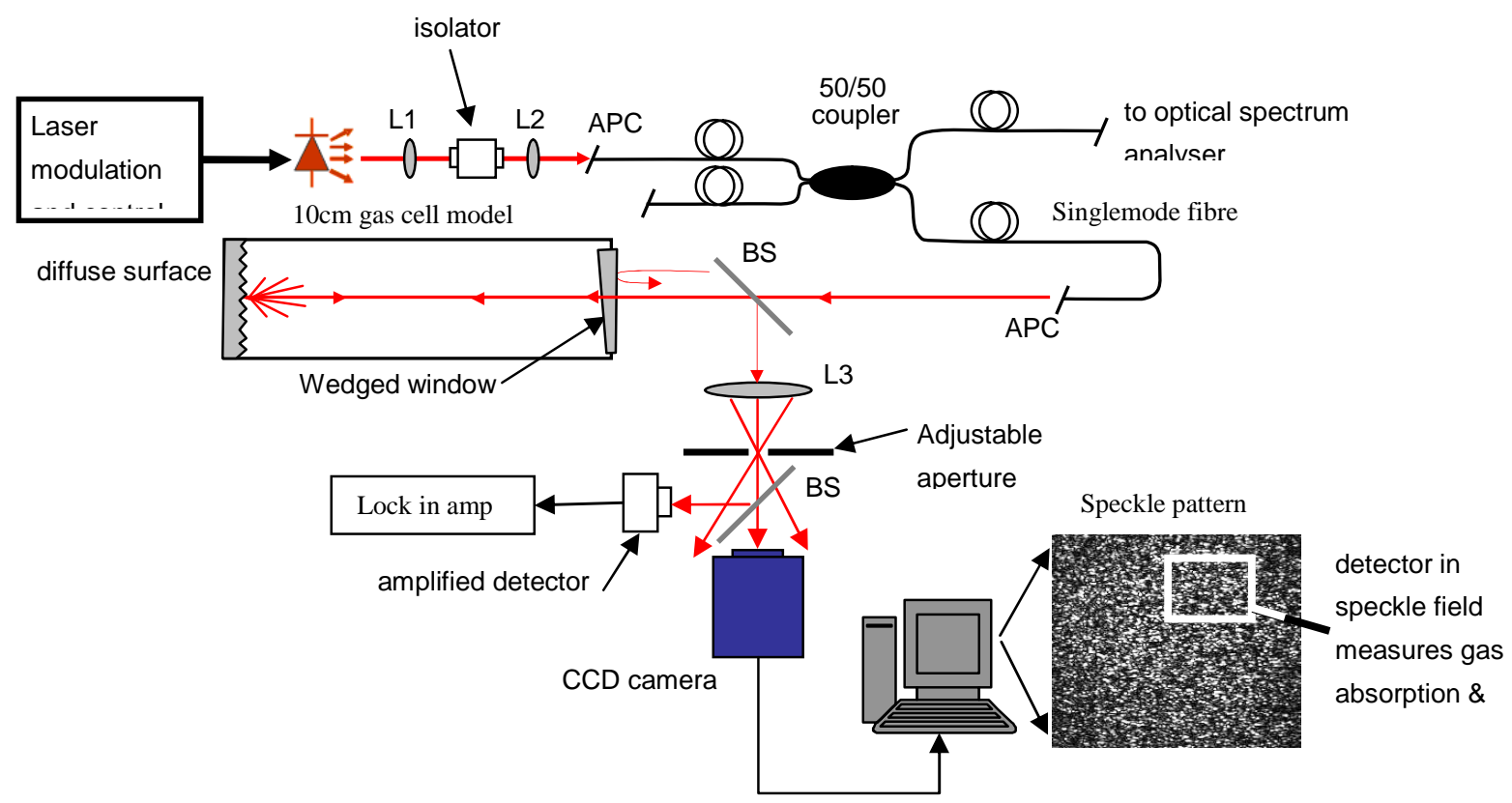

Figure 4. Experimental setup for investigating the use of diffuse reflections in WMS. Key, L1, L2, L3; lens, APC; angle polished connector, BS; beam splitter.

\subsection{Experimental confirmation of speckle behaviour}

We conducted a number of experiments described below to verify that our configuration was valid for conducting speckle related investigations.

\subsubsection{Speckle size.}

By varying the diameter of the adjustable aperture in Figure 4 and observing the speckle field formed, we confirmed that the lateral speckle size of the subjective speckle was related to the F\# according to equation (2). Speckle fields corresponding to aperture diameters of $1 \mathrm{~mm}$ and $0.6 \mathrm{~mm}$ are shown in Figure 5 .

\subsubsection{Interferometric speckle.}

The window on gas cell model in Figure 4 could be aligned such that the object beam (scattered light from the diffuser) and the reference beam (specular reflection from the window) combined coherently to form an interferogram (interferometric speckle) or misaligned such that only the object beam (noninterferometric speckle) was imaged by the lens onto the CCD camera. We confirmed that we could obtain interferometric speckle with our configuration by performing a technique commonly referred to as static frame subtraction in Electronic Speckle Pattern Interferometry (ESPI)[17]. In subtraction 
ESPI the interferogram from an object in its initial state is recorded and stored electronically. The object is then displaced and the digitized live interferogram of the deformed state of the object is subtracted pixel by pixel from the stored signal. Areas of the two images where the speckle pattern remains in phase will give a resultant signal of zero, while out of phase areas will give non-zero signals[14]. Correlation ESPI fringes obtained with the experimental set up shown in Figure 4 are shown in Figure 5(c). These were obtained by heating the diffuse surface (metallic surface covered with retroreflective tape) and then performing static frame subtraction as the diffuse object cooled down. In subtraction ESPI, correlation fringes can only be obtained in the case of interferometric speckle.

(a)

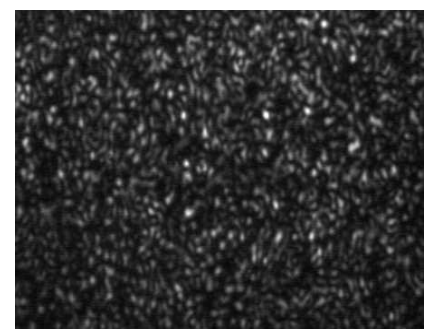

(b)

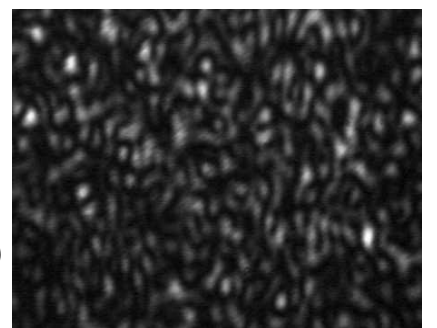

(c)

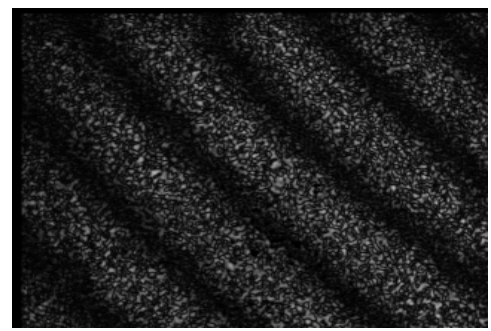

Figure 5. (a) Small speckles corresponding to a large aperture $(1 \mathrm{~mm})$, (b) large speckles $(0.6 \mathrm{~mm}$ aperture), (c) correlation fringes obtained by performing subtraction ESPI using speckles larger than the CCD pixels.

\subsection{Statistics of practical speckle}

The speckle theory outlined in section 2 is based on the assumption that the surface height deviations are large in comparison to the wavelength of the radiation illuminating it, and that the resultant speckle field is completely polarised. In general surfaces at which polarised light is singly scattered give rise to polarised speckle, this includes lightly-scattering transmission elements, such as ground glass. Bulk diffusers (surfaces into which light penetrates and is multiply scattered) on the other hand, such as matt white paint or opal glass, depolarise the light and thus do not generate a fully developed speckle pattern $^{[13]}$. The statistics of speckle from surfaces with height deviations less than the illuminating wavelength have been shown to depend on the surface roughness[18]. The purpose of this section is to investigate the possibility of exploiting the characteristics of under developed speckle as a means of reducing the speckle related intensity uncertainty.

We investigated a number of diffusely scattering materials including PTFE (polytetrafluoroethylene), Macor (white machinable ceramic), ground glass, opal glass, spectralon ${ }^{\mathrm{TM}}$ (a PTFE based material made by Labsphere), metallic surfaces painted with reflective paint (Reflect made by Rocol) and retroreflective tape. Due to different levels of scattering and depolarisation in the different samples the speckle fields are expected to exhibit various levels of development $(0<\mathrm{C}<1)$. The speckle patterns 
generated by the various samples were characterised by plotting probability density function, contrast and observing the size of the speckle. For these experiments we used a HeNe laser emitting at $632.8 \mathrm{~nm}$ for convenience. The experimental setup is shown in Figure 6 (only the reflective geometry is illustrated).

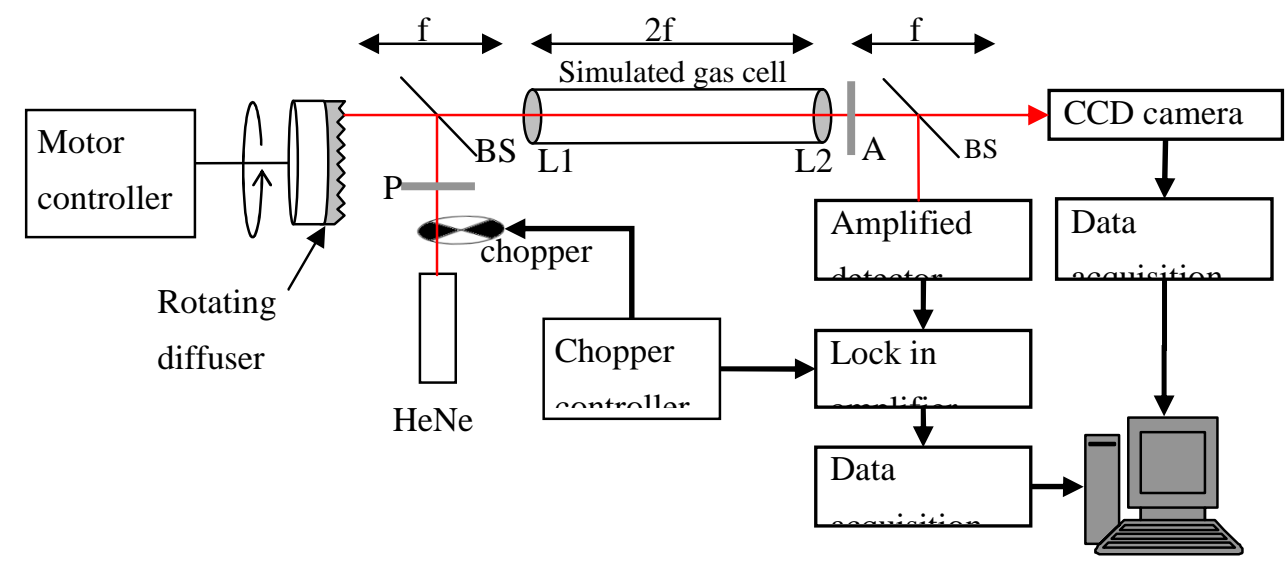

Figure 6. Experimental setup for characterising properties of speckle from various scattering materials. Key: P; polariser, A; analyser, BS; beam splitter, f; focal length of lens L1 and L2.

Light from a linearly polarised HeNe was directed onto the target samples. Transmitted or reflected light was then directed either to a detector or a CCD camera after passage through the gas cell model. Lenses were used as gas cell windows; L1 collimated the scattered light and L2 focussed the light on to the detector. The detector signal was passed to a lock in amplifier that was locked to the chopper frequency.

\subsubsection{Dependence of speckle statistics on surface roughness.}

Fujii and Asakura ${ }^{[18]}$ have investigated the effect of surface roughness on the statistical distribution of image speckle intensity. They have shown that the probability distribution and the standard deviation of the intensity fluctuations in image speckle patterns are strongly dependent on the surface roughness h. The probability distribution was found to vary from a negative exponential distribution for surfaces with $\mathrm{h}>\lambda$ to a blend of Poissonian and Gaussian distributions for decreasing value of roughness. They similarly found that the standard deviation of the intensity fluctuations increases almost linearly with increasing roughness and then levels off at a constant value. This constant value corresponds to the fully developed speckle condition. We captured images of speckle patterns from various samples obtained under identical conditions to confirm the dependence of speckle size on surface roughness. The images were captured with L2 (Figure 6) removed. For most of the samples (Macor, spectralon ${ }^{\mathrm{TM}}$, spray painted surface, retroreflective tape), there were no visible differences in speckle size. However PTFE gave rise to visibly smaller speckle. The speckle images from the PTFE and spectralon ${ }^{\mathrm{TM}}$ are shown in Figure 7. The images were caputerd with lens L1 operating at F\# $=2.6$ and with a static 
diffuser. This appears to suggest that speckle related intensity uncertainty can be reduced by using diffusers with surface height deviations less than the wavelength of the illuminating light. In future we plan to carry out a systematic investigation of the last point by measuring the surface roughness of our samples and using computational techniques for estimating speckle size.
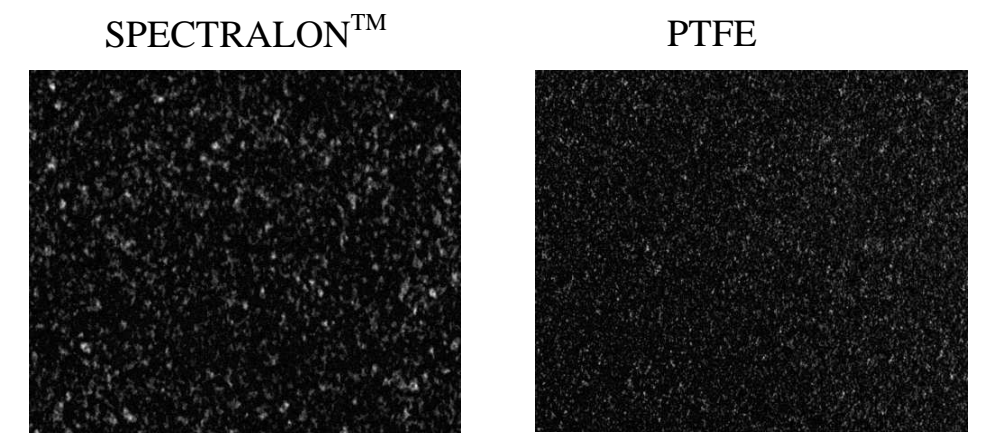

Figure 7. Dependence of speckle size on surface roughness. (a) SPECTRALON ${ }^{\mathrm{TM}}(460 \times 460$ pixels), (b) $10 \mathrm{~mm}$ thick PTFE $(460 \times 460$ pixels $)$

\subsubsection{Degree of polarisation (DOP).}

Light emerging from a scattering medium illuminated by linearly polarised light consists of linearly and randomly polarised photons ${ }^{[19]}$. This is directly related to the number of scattering events experienced by the photons. In section 4.2.2 we demonstrated interference effects by coherently adding a speculary reflected beam to a speckle pattern from a surface scatterer. This was possible because the speckle pattern had a high DOP (degree of polarisation). From a gas sensing point of view depolarised speckle is preferable for several reasons. Firstly, depolarised speckle is under developed, therefore we expect the contrast to be less than unity. The argument for this has been summarised by Ennos $^{[13]}$ as follows; "since the speckle phenomenon is itself an interference one, a true 'fully developed' speckle pattern can derive only from interference of light that is all polarised in the same manner". Secondly, depolarised speckle will produce reduced visibility of interference effects if it combines with specular reflections from elsewhere in the optical system in comparison to polarised speckle. Thirdly, polarization effects have been shown to affect the number of speckles integrated by the detector with the maximum number of speckles occurring when the incident light is completely depolarised by the diffuse reflector ${ }^{[20]}$. Therefore, in addition to speckle size and detector size, the number of speckles integrated by a detector also depends on the amount of depolarization from the diffuse scatterer. We conducted an experiment to measure the DOLP (degree of linear polarisation) of scattered light from the various samples. The DOLP is given by ${ }^{[19]}$

$$
D O L P=\frac{I_{P}-I_{C}}{I_{P}+I_{C}}
$$

where $I_{p}$ and $I_{c}$ are the mean intensities measured with parallel and crossed polarisers respectively ${ }^{[20]}$. The results are presented in Table 1 below. 
Table 1. Degree of linear polarisation of light reflected or transmitted from various samples

\begin{tabular}{|c|c|c|}
\hline MATERIAL & $\begin{array}{l}\text { DEGREE OF } \\
\text { LINEAR } \\
\text { POLARISATION }\end{array}$ & CONTRAST \\
\hline \multicolumn{3}{|l|}{ REFLECTIVE } \\
\hline 10mm thick PTFE & 0.02 & 0.98 \\
\hline spectraflect $^{\mathrm{TM}}$ & 0.07 & 1.20 \\
\hline $20 \mathrm{~mm}$ thick spectralon ${ }^{\mathrm{TM}}$ & 0.13 & 1.00 \\
\hline $\begin{array}{l}\text { aluminium surface painted } \\
\text { with a reflective paint }\end{array}$ & 0.78 & 1.10 \\
\hline retroreflective tape & 0.9 & 1.00 \\
\hline \multicolumn{3}{|l|}{ TRANSMISSIVE } \\
\hline opal glass & 0.2 & 1.00 \\
\hline ground glass & 1 & 1.00 \\
\hline
\end{tabular}

The DOLP was calculated from the measurements of $I_{p}$ and $I_{c}$ that were obtained with parallel and crossed polariser $(\mathrm{P})$ and analyser $(\mathrm{A})$ pair respectively (Figure 6). The polarisers were only used for this experiment. As expected the surface scatters exhibit a high DOLP compared to the bulk or volume scatters.

\subsubsection{Integrated and blurred speckle.}

In practice speckle intensity measurements are made with detectors with finite apertures. As a result the measured intensity is an integrated version of the ideal point detector. Relative motion between the detector and the scattering surface can also lead to blurring of the speckles leading to reduction in contrast. The first-order statistics of integrated and blurred speckles have been considered by Goodman $^{[21]}$. He has shown that for a non stationary speckle pattern, the mean of the measured intensity is equal to the true mean of the static pattern. Adding M independent speckle patterns has been shown to reduce the uncertainty by $\sqrt{M}^{\text {[20] }}$.

Statistically uncorrelated speckle patterns were generated by capturing ten images (set 1) whilst the diffuser was rotated very slowly using the experimental setup of Figure 6. From these images a set of nine images were generated by computing the average of $K$ images where $K=2,3 \ldots . .10$. We then calculated the contrast of the average images. We also took ten images of the diffuser whilst stationary (set 2) at arbitrary time intervals between 30 seconds and 120 seconds. These were also subjected to the same processing as set 1 . Figure 8 shows the averaged images and the plots of their corresponding probability density functions. Also shown in Figure 8 are the 3D representations of the intensity distribution corresponding to one speckle pattern and the average of ten speckle patterns. The plot of contrast against number of images averaged for the two sets of images is shown in Figure 9. A blurred speckle image speckle image was also captured whilst the diffuser was rotated very quickly (Figure $10)$. 
one speckle pattern

(a1)

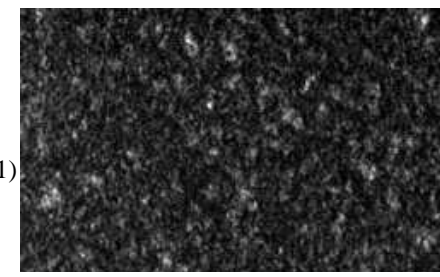

PDF of one pattern, image (a1)

(a2)

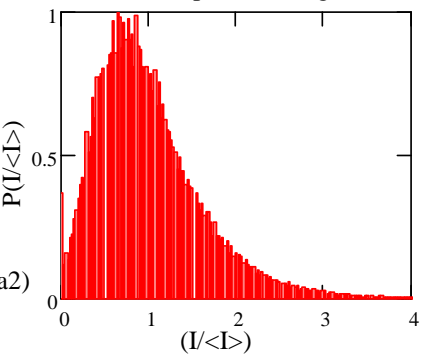

(a3)

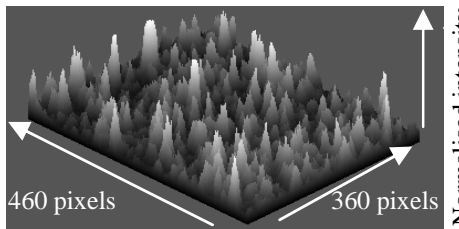

$3 \mathrm{D}$ representation of the average of one speckle pattern, image (a1) average of two speckle patterns

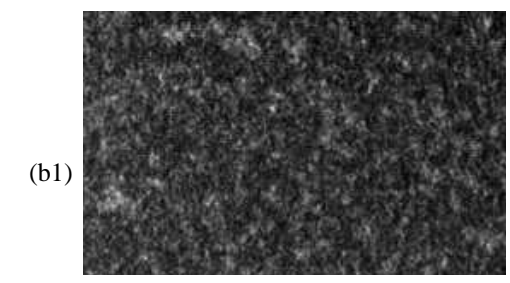

PDF of two speckle patterns, image (b1)
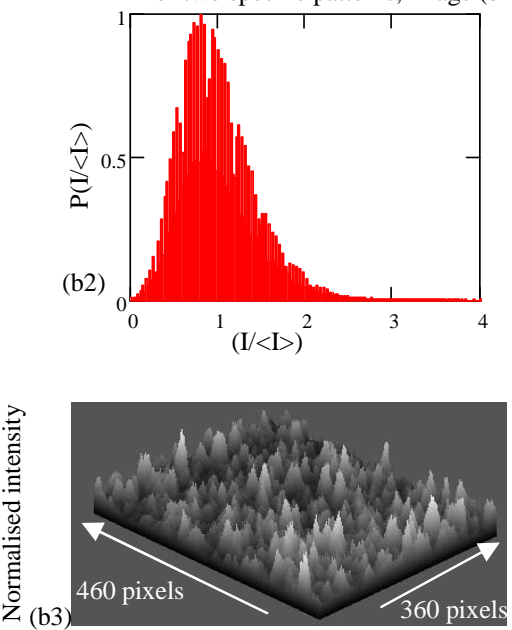

3D representation of the average of two speckle patterns, image (b1) average of ten speckle patterns

(c1)

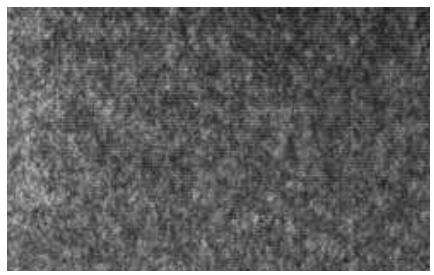

PDF of ten speckle patterns image (c1)

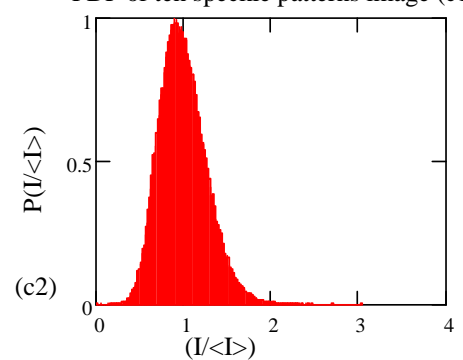

c3)

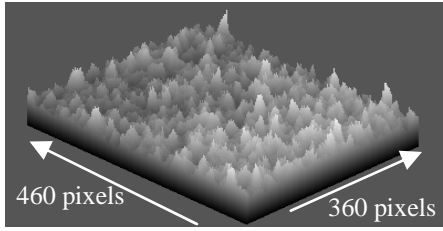

$3 \mathrm{D}$ representation of the average of ten uncorrelated speckle patterns, image (c1)

Figure 8. (a1), (b1) and (c1) are images (460 $\times 360$ pixels) of speckle patterns; (a2), (b2), (c2) the associated probability density functions PDFs, (a3), (b3) and (c3) are 3D representations of images (a1), (b1) and (c1) respectively.

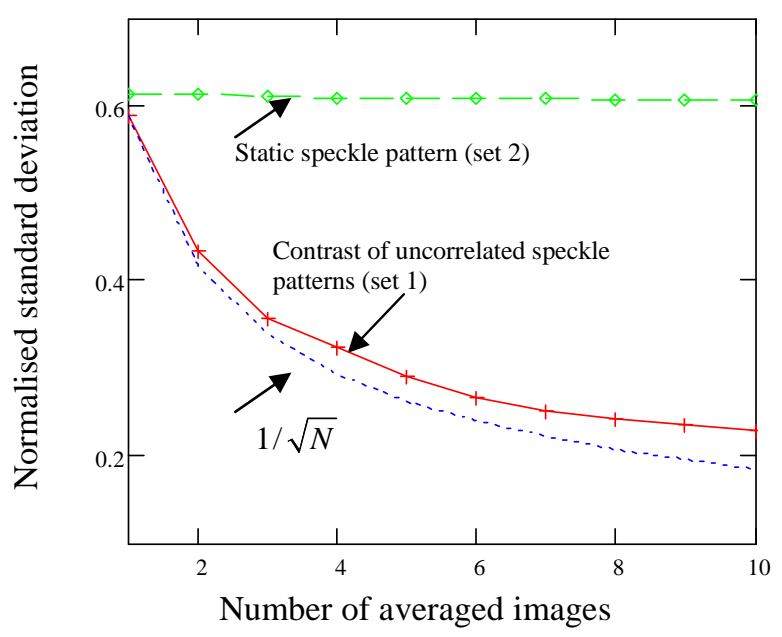

Figure 9. Variation of contrast or normalised standard deviation with the number of images averaged.

Set 1; uncorrelated speckle patterns, Set 2; images taken from a static diffuser. 
(a)

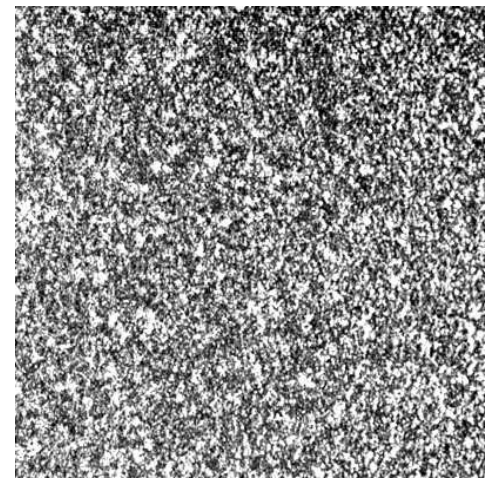

(b)

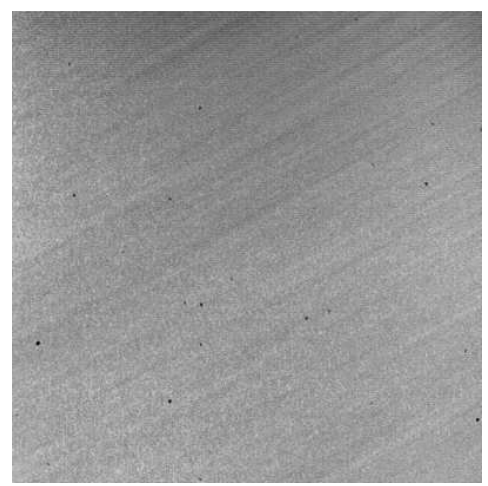

Figure 10. Blurring speckle using a moving diffuser. (a) image of a static diffuser, (b) image taken whilst rotating the diffuser

\section{Effect of wavelength modulation on speckle.}

We investigated the level of uncertainty that relates to wavelength modulation by considering an intensity measurement made at one wavelength $\lambda$ with a second measurement made within a short modulation period, at a second wavelength $(\lambda+\delta \lambda)$. We examined the effect of the wavelength modulation on interferometric and non-interferometric speckle on a scale relevant to gas detection using direct spectroscopy wavelength modulation spectroscopy.

\subsubsection{Effect of wavelength modulation on non-interferometric speckle.}

For non-interferometric speckle, the expected change in the speckle field due to wavelength change can be estimated by evaluating (4) at $\lambda_{1}$ and $\lambda_{1}+\delta \lambda$. For example, substituting $\mathrm{F} \#=2.5, \lambda_{1}=1650 \mathrm{~nm}$ and $\delta \lambda=10 \mathrm{GHz}$ in (4), the proportional change in speckle related uncertainty is $6 \times 10^{-5}$. This level of uncertainty is relevant to differential measurements or when scanning across the gas line to obtain the harmonic line shapes or for direct spectroscopy. For line locked WMS with a residual frequency jitter of $10 \mathrm{MHz}$ the proportional change in speckle related uncertainty is $6 \times 10^{-8}$. The speckle related uncertainty due to wavelength change of non-interferometric speckle is below the level of a static speckle field.

\subsubsection{Effect of wavelength modulation on interferometric speckle.}

Interferograms (a polarised speckle pattern combined with a smooth reference wave or a another speckle pattern) obtained at different wavelengths have a phase difference that is associated with the wavelength change. This applies only if the two interferograms remain spatially correlated in the measurement interval. Sirohi[17] has shown that the phase difference $\delta \phi$ created between such interferograms is equal to 


$$
\delta \phi=2 \pi L \delta \lambda / \lambda^{2}
$$

where in our case, $L$ is the physical path difference between the wedged window and the scattering surface in Figure 4 . The value of $\delta \phi$ determines whether the observed speckle fields are in phase or out of phase, with the correlation coefficient being given by[17]

$$
\rho(\delta \phi)=(1+\cos \delta \phi) / 2
$$

Thus, for increasing $\delta \phi$, the speckle fields alternate between $\rho(\delta \phi)=1$ (in phase) and $\rho(\delta \phi)=0$ (out of phase). If the speckle fields are in phase, we expect the speckle-related noise to reduce to zero for differential measurements. We conducted an experiment to obtain a plot of the correlation of interferograms corresponding to different wavelengths. We used a technique that was employed by Olszak and Tatam[22] to calibrate the path-length imbalance in optical fibre ESPI systems. We used the experimental setup of Figure 4.

The specular reflection from the wedged window was used as the reference smooth wave. The method adopted was to store a reference interferogram for an initial injection current or wavelength value. The wavelength was scanned by a ramping the injection current, causing a corresponding change in the phase. New interferograms were acquired continuously. The subsequent images were then subtracted from the reference image pixel by pixel, the result rectified and the average intensity of the resultant image calculated. A plot of average intensity against current is shown in Figure 11.

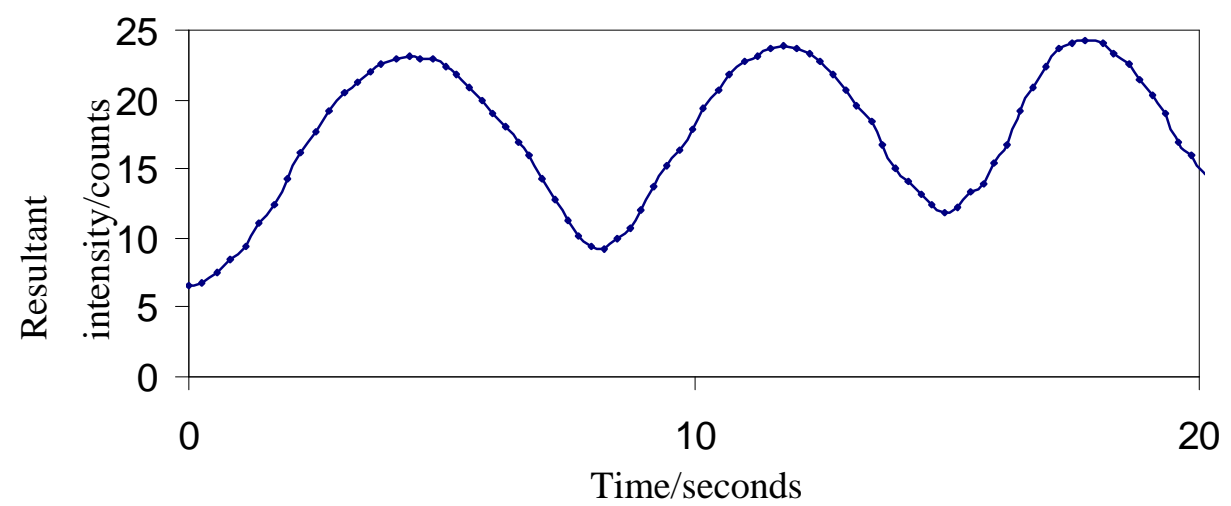

Figure 11. A plot average intensity that maps the phase change of the interferometer with wavelength. The troughs correspond to $\rho(\delta \phi)=1$ (in phase) and the peaks correspond to $\rho(\delta \phi)=0$ (out of phase).

The plot is on a sloping background due to simultaneous wavelength and intensity modulation. The period of the ramp waveform was 100 seconds. This allowed a sufficient number of frames to be captured in the interval $0<\delta \phi<\pi$. When the phase shift equalled $\pi$ the average intensity attained its maximum value and when it was $2 \pi$ the average intensity was a minimum; that is, subtracting from a speckle image an identical speckle image results in a 'black' (zero-intensity) image. Approximately 16 maxima could be seen in a $26.6 \mathrm{GHz}$ interval, yielding an etalon spacing of $9.2 \mathrm{~cm}$. The length of the 
simulated gas cell was $9 \mathrm{~cm}$. These observations suggest that the fringes originate from the low finesse cavity formed by the simulated gas cell. This shows that an interference fringe like signal can occur due to wavelength modulation of interferometric speckle.

\section{Implications of speckle related uncertainty for TDLAS}

In this section we will consider the implications of the non-interferometric and interferometric speckle related uncertainty on direct spectroscopy and wavelength modulation spectroscopy using the gas cell model illustrated in Figure 4. We simulated the aforementioned techniques by driving the laser diode appropriately. The live camera speckle images were simultaneously recorded and the individual frames were saved as sequential bitmap files or an AVI (audio video interleave) file.

\subsection{Direct spectroscopy}

In direct spectroscopy the laser is tuned over the absorption line by either ramping the operating current or temperature. The principle of direct spectroscopy is illustrated in Figure 1. We simulated direct spectroscopy by scanning the laser frequency over a $26 \mathrm{GHz}$ range. This was achieved by modulating the operating current with a low frequency ramp signal whilst continuously recording the speckle images and saving them to an AVI file. A number of programs were written in Labview ${ }^{\mathrm{TM}}$ to read and process AVI files. One program converted the image frames into an array of individual pixel data. The array was then divided the array into grids (pixel binning) representing our detection system. We could choose the size of the bins to match the size of our detectors or any arbitrary size (see Figure 12). Another program divided the image array into ten bins of user selectable size and origin (see Figure 12(b))

(a)

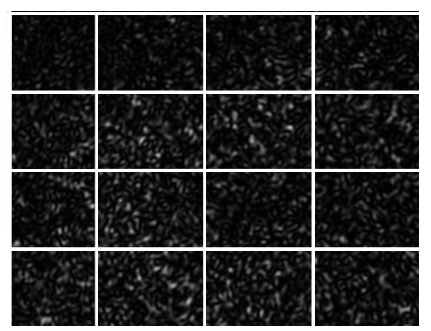

(b)

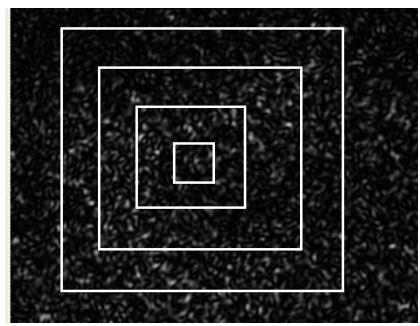

Figure 12. Illustration of the different types of pixel binning that were employed. (a) images were divided into equal bins size bins equivalent to detector size (b) different size bins used to evaluate the effect of averaging different number of pixel. 


\subsubsection{Non-interferometric speckle.}

In section 5.1.1, it was established that the speckle related intensity uncertainty of non-interferometric speckle due to wavelength modulation is below the level of a static field. Therefore the level of noise in this case will be similar to that of a static field. We examined the uncertainty of a static field by considering the uncertainty associated with placing a detector of a particular size in different areas of the speckle field by using the binning method illustrated in Figure 12(a). For a given bin (or detector) size the speckle related measurement uncertainty was calculated from the mean intensity of each bin and the standard deviation of the mean intensities of all the bins. The exercise was done for various bin sizes and two different speckle sizes. We used the images shown in Figure 7. The results are presented in Table 2. As expected, for small areas the uncertainty is higher.

Table 2. Speckle related intensity noise for various detector sizes.

\begin{tabular}{|c|c|c|c|c|c|c|c|}
\hline & & \multicolumn{3}{l|}{ SPECTRALON } & \multicolumn{2}{l|}{ PTFE } \\
\hline $\begin{array}{c}\text { detector or } \\
\text { bin size } \\
(\text { pixels })\end{array}$ & $\begin{array}{c}\text { approximate } \\
\text { bin area } \\
\left(\mathrm{mm}^{2}\right)\end{array}$ & mean & $\begin{array}{c}\text { Standard } \\
\text { deviation }\end{array}$ & $\begin{array}{c}\text { proportional } \\
\text { Standard } \\
\text { deviation }\end{array}$ & mean & $\begin{array}{c}\text { standard } \\
\text { deviation }\end{array}$ & $\begin{array}{c}\text { proportional } \\
\text { Standard } \\
\text { deviation }\end{array}$ \\
\hline $40 \times 40$ & 0.25 & 19.38 & 3.5 & 0.18 & 19.41 & 2.8 & 0.14 \\
\hline $80 \times 80$ & 1 & 19.76 & 2.8 & 0.14 & 19.41 & 2.5 & 0.13 \\
\hline $120 \times 120$ & 2.2 & 19.38 & 1.8 & 0.093 & 19.29 & 2.2 & 0.11 \\
\hline $160 \times 160$ & 4 & 19.10 & 1.2 & 0.063 & 18.96 & 1.3 & 0.07 \\
\hline
\end{tabular}

\subsubsection{Interferometric speckle.}

We scanned the laser frequency with a ramp signal with a period of $10 \mathrm{~s}$ and saved the frames that were continuously recorded to an AVI file. The AVI file was processed using the binning method illustrated in Figure 12(b). The average intensity of each bin for all the frames was computed and plotted as a graph of average intensity against time corresponding to the signal that would be recorded by a detector of a given size placed in a particular position. We observed interference fringes superimposed on the ramp signal that was used to scan the laser frequency. We investigated the effect of detector size on the visibility of the fringes. We varied the area over which the speckles were averaged by using the binning method illustrated in Figure 12(b). The intensity of each pixel varies as (7), however the phases of the pixels are randomly distributed. Therefore by averaging a sufficient number of pixels, a reduction in the visibility of the fringes is expected. Shown in Figure 13 are the plots obtained for detector or bin sizes comprising of one pixel and a hundred pixels. The approximate maximum peak to peak intensity excursions for the different averaging areas are shown in Table 3. 


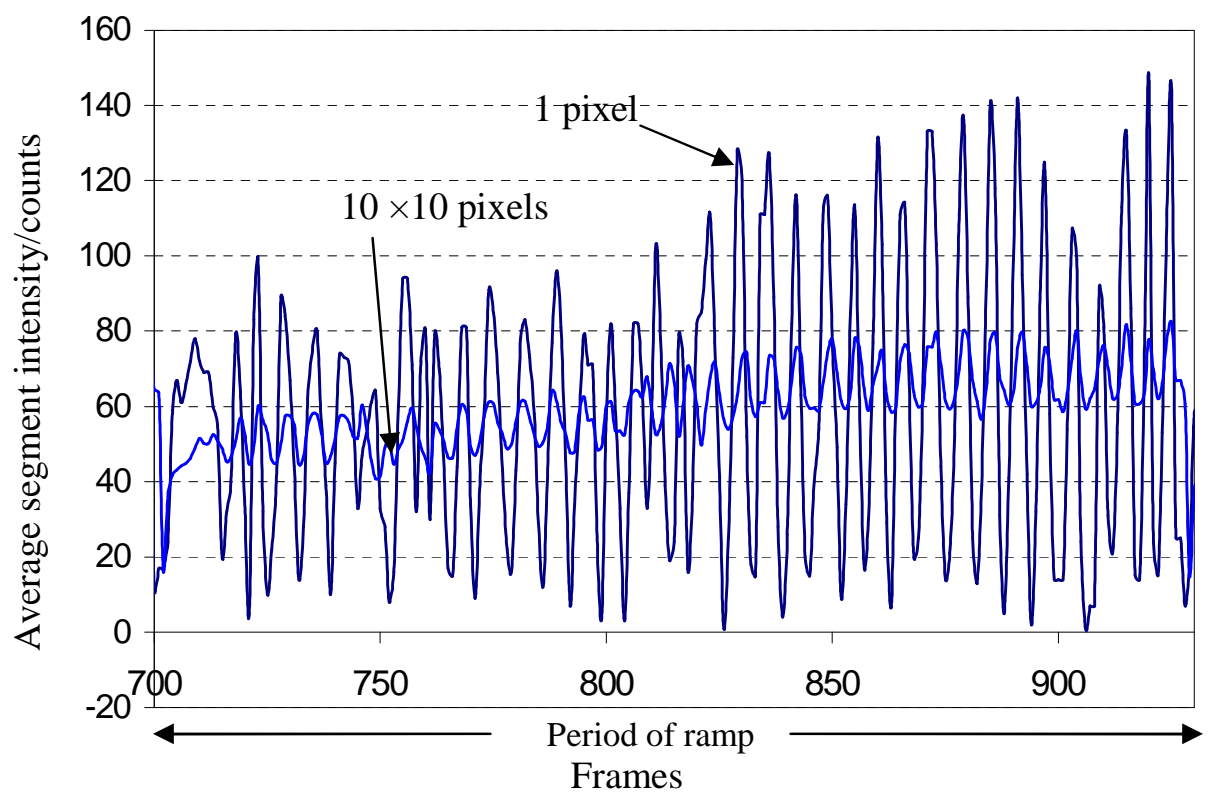

Figure 13. Reduction in the visibility of the interference fringes with increasing modelled detector size. Fringes were formed by modulating interferometric speckle. After a certain detector size, further increase of the detector size yields no significant reduction in the amplitude of the interference fringes

Table 3. Approximate peak to peak amplitude of the intensity modulation for different area averaging size

\begin{tabular}{|r|r|r|}
\hline $\begin{array}{r}\text { Detector or bin size } \\
\text { (pixels) }\end{array}$ & $\begin{array}{r}\text { Approximate pixel area } \\
\left(\mathrm{mm}^{2}\right)\end{array}$ & $\begin{array}{r}\text { Maximum peak to peak intensity excursion } \\
\text { (counts) }\end{array}$ \\
\hline 1 & $1.6 \times 10^{-4}$ & 153 \\
\hline $3 \times 3$ & $1.4 \times 10^{-3}$ & 127 \\
\hline $5 \times 5$ & $3.9 \times 10^{-3}$ & 75 \\
\hline $7 \times 7$ & $7.6 \times 10^{-3}$ & 20 \\
\hline $10 \times 10$ & 0.016 & 14 \\
\hline $40 \times 40$ & 0.25 & 12 \\
\hline $80 \times 80$ & 1 & 6 \\
\hline $120 \times 120$ & 2.2 & 4 \\
\hline $160 \times 160$ & 4 & 4 \\
\hline $200 \times 200$ & 6.2 & 4 \\
\hline
\end{tabular}




\section{Gas absorption measurements}

An experiment was conducted to confirm the dependency of the speckle related uncertainty on the number of speckles integrated by the detector for the case of non-interferometric speckle. A calibrated variable aperture was placed in front of a large area detector $\left(19.6 \mathrm{~mm}^{2}\right)$ and methane gas concentration was measured at 3 different aperture sizes. The experimental set up is described below.

\subsection{Experimental setup for Gas absorption measurements}

Modulated light from a custom DFB (distributed feed back) laser diode (configuration similar to van Well et al[23]) was coupled into a single mode fibre circulator (FiberLogix FCIR-65-2-L-1) with angle polished connectors through an antireflection coated aspheric lens. The DFB laser was driven by a current controller (Thorlabs LDC 202) and a temperature controller (Thorlabs TED 200). The modulation of the DFB current was achieved by supplying the current controller with a $6 \mathrm{kHz}$ sine waveform from a signal generator (Hewlett Packard HP33120A). This waveform was also supplied to lock in amplifiers (Stanford Research Systems SR850) as a reference signal.

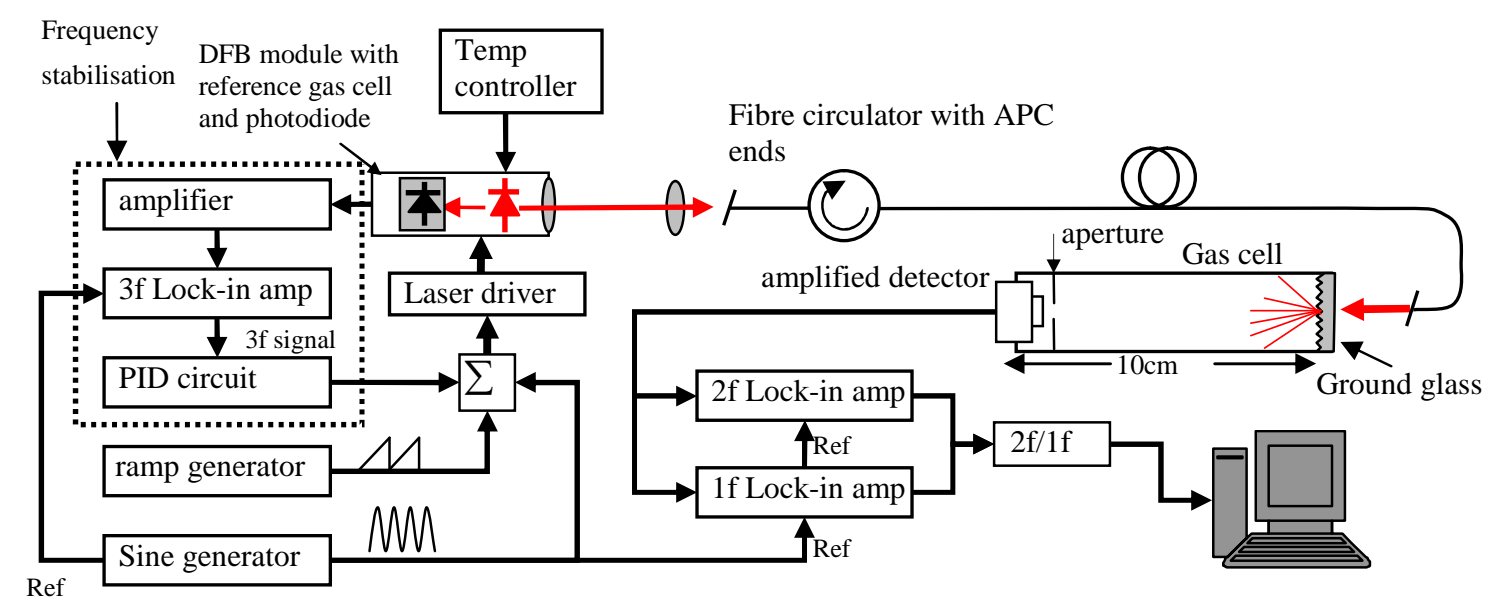

Figure 14. The experimental setup for methane gas detection using WMS. Key: SA, spectrum analyser; PID, proportional-integral-differential; APC, angle polished connector, Ref; reference signal supplied to the lock in amplifiers

The output light was directed onto a large area $\left(19 \mathrm{~mm}^{2}\right)$ amplified detector (Thorlabs PD50B) after passage through a $10 \mathrm{~cm}$ long gas cell with a ground glass front window`. The output of the detector amplifier was fed to 2 lock in amplifiers (Stanford Research Systems SR850) for phase sensitive detection at $6 \mathrm{kHz}(1 \mathrm{f})$ and $12 \mathrm{kHz}(2 \mathrm{f}$ ). The $2 \mathrm{f}$ signal was then normalised by the $1 \mathrm{f}$ signal. Both the $2 \mathrm{f}$ and 1f signals are directly proportional to the incident intensity. Therefore dividing one by the other 
removes this incident power dependency. The normalised $2 \mathrm{f}$ signal was transferred to a computer running Labview ${ }^{\mathrm{TM}}$ software through data acquisition card (National Instruments PCI 6259)

Within the DFB module, light from the rear facet of the DFB is falls onto a Ge photodiode that is enclosed in a standard TO can containing $100 \%$ methane. This served as a reference methane gas cell. The photodiode signal was amplified by a transimpedance amplifier $(100 \mathrm{k} \Omega$ gain) before being fed to a lock in amplifier (Stanford Research Systems SR850) where it was demodulated at $18 \mathrm{kHz}$. The $3 \mathrm{f}$ signal was used by a feed back control circuit for locking the laser frequency to the peak of the $2 \mathrm{f}$ gas absorption line.

\subsection{Gas measurements with different detector size}

A calibrated variable aperture was placed in front of the large area detector $\left(19 \mathrm{~mm}^{2}\right.$ active area). Methane gas concentration was measured at 3 different aperture sizes (diameters; $1 \mathrm{~mm}, 3 \mathrm{~mm}$ and $12 \mathrm{~mm}$ ). Methane ( $2.5 \%$ concentration) was gradually diluted with hydrocarbon free air using gas flow controllers (Telydyne THPS-400-230). The peak $2 \mathrm{f}$ signal normalised by the $1 \mathrm{f}$ signal was recorded for different concentrations; from an initial concentration of 1000ppm down to 25ppm (ppm, parts per million). The results for measurements with the $3 \mathrm{~mm}$ and $12 \mathrm{~mm}$ apertures placed in front of the detector are shown in Figure 15 and Figure 16 respectively.

\section{Results}

At concentrations below 125ppm; gas absorption measurements with the $1 \mathrm{~mm}$ aperture were indistinguishable from background signals and it can be seen from the inserts in Figure 15 and Figure 16 that measurements made with the $3 \mathrm{~mm}$ aperture have a higher associated uncertainty than those made with the $12 \mathrm{~mm}$ aperture placed in front of the detector. 


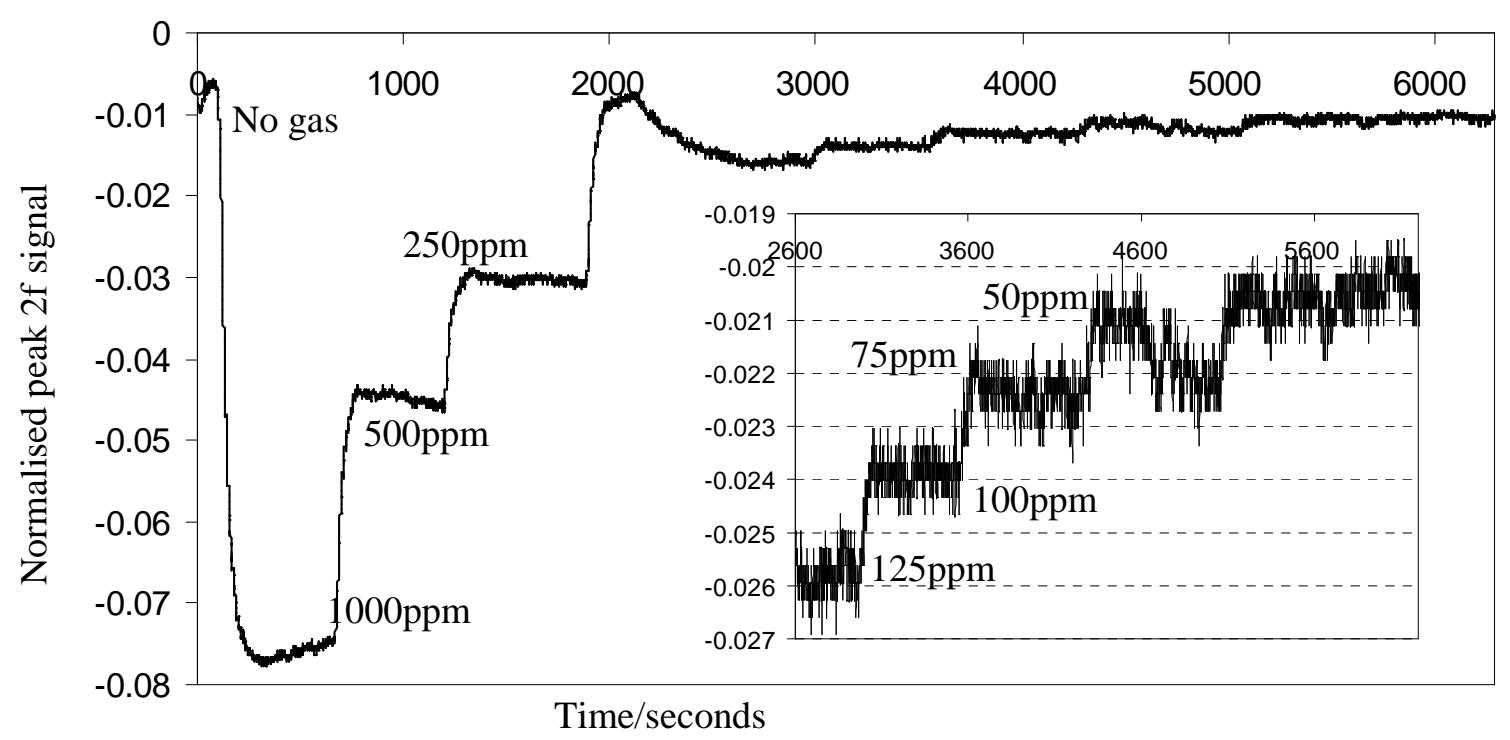

Figure 15. Gas concentration measurements with a $3 \mathrm{~mm}$ aperture placed in front of the detector. Insert; shows the response for concentrations below 125ppm. Key, ppm; parts per million

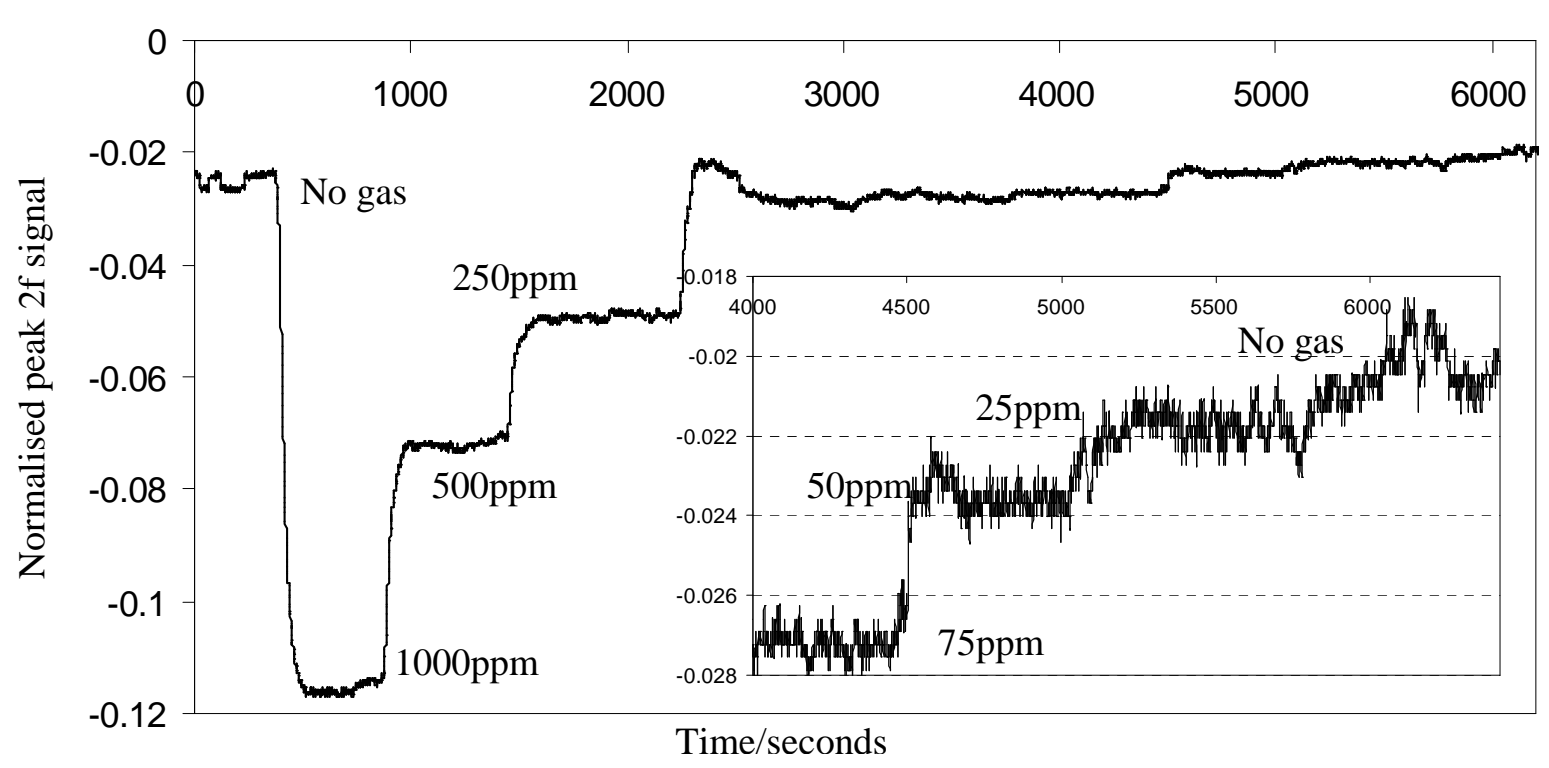

Figure 16. Gas concentration measurements with a $12 \mathrm{~mm}$ aperture placed in front of the detector. Insert; shows the response for concentrations below 75ppm. Key, ppm; parts per million

\section{Discussion and Conclusion}

Methods that have been proposed to reduce the effects of interference fringes include careful alignment of optical components or mechanically jittering the offending components and post 
detection processing. Evidence also suggests that in the right circumstances use of diffusely scattering materials may reduce fringes. We have investigated their use and the consequent introduction of random uncertainty associated with generation of laser speckle. We have developed a methodology for investigating the associated noise and presented preliminary data for confirmation of the principle.

Future work will involve; testing of our predictions, investigating diffusely reflecting materials that have surface roughness less than the wavelength of the illuminating light and investigating different gas cell geometries including integrating spheres.

\section{Acknowledgements}

This work is supported by the Engineering and Physical Sciences Research Council (EPSRC), UK under grant No.GR/T04601/01. Jane Hodgkinson is supported by an EPSRC Advanced Fellowship, GR/T04595/01.

\section{References}

1. Silver, J. A. and Stanton, A. C., Appl. Opt. 27, 10 (1988)

2. Schiff H I, Mackay and Bechara J Air Monitoring by Spectroscopic Techniques Editor Sigrist M W (New York: John Wiley and sons) pp 239-333.

3. McManus, B. J and Kebabian, P. L., Appl. Opt. 29, 7 (1990)

4. Mansfield, C. D. and Rutt, H. N., Measurement Science and Technology 10, 3 (1999)

5. Webster, C. R., J. Opt. Soc. Amer. B 2, (1985)

6. Chou, S., Baer, D. S., and Hanson, R. K., Appl. Opt. 36, 15 (1997)

7. Reid, J., El-Sherbiny, M., Garside, B. K., and Ballik, E. A., Appl. Opt. 19, (1980)

8. Sano, H., Koga, R., and Kosaka, M., Jpn. J. Appl. Phys., Part 1 22, 12 (1983)

9. Iguchi, T., J. Opt. Soc. Am. B: Opt. Phys. 3, 3 (1986)

10. Capellani F, Mellandrone G and Restelli G Monitoring of Gaseous Pollutants by Tunable Diode Lasers editors Grisar R, H. Preier H, Schmidtke G and Restelli G (UK: Kluwer Academic Publishers) pp 51-60.

11. Sun, H. C and Whittaker, E. A., Appl. Opt. 31, 24 (1992)

12. Tranchart, S, Bachir, I. H., and Destombes, J-L., Appl. Opt. 35, 36 (1996)

13. Ennos A E Laser Speckle and Related Phenomena : Springer-Verlag) pp 203-53.

14. Holographic and Speckle Interferometry $2^{\text {nd }}$ edn Jones $\mathrm{R}$ and Wykes $\mathrm{C}$ (Cambridge, UK: Cambridge University Press).

15. Goodman, J. W., J. Opt. Soc. Am. 66, 11 (1976)

16. Werle, P., Spectrochimi. Acta Part A 54, (1998)

17. Sirohi, R. S., Contemporary Physics 43, 3 (2002) 
18. Fujii H and Asakura T, Opt. Commun. 111 (1974).

19. Morgan, S. P. and Ridgway, M. K., Opt. Express. 7, 12 (2000)

20. MacKerrow, E. P. and Schmitt, M. J., Appl. Opt 36, 27 (1997)

21. Goodman J W Laser Speckle and Related Phenomena : Springer-Verlag) pp 9-75.

22. Olszak, A. and Tatam, R. P., Meas. Sci. Technol. 8, 7 (1997)

23. van Well, B., Murray, S., Hodgkinson, J., Pride, R., Strzoda, R., Gibson, G., and Padgett, M., J Opt. A-Pure Appl. Opt, 7, (2005) 